\title{
A bound on the superpotential
}

\section{Michael Dine, ${ }^{a}$ Guido Festuccia ${ }^{a}$ and Zohar Komargodski ${ }^{b}$}

${ }^{a}$ Santa Cruz Institute for Particle Physics, University of California Santa Cruz, 1156 High street, Santa Cruz, CA 95064, U.S.A.

${ }^{b}$ School of Natural Sciences, Institute for Advanced Study, Einstein Drive, Princeton, NJ 08540, U.S.A.

E-mail: dine@scipp.ucsc.edu, guido@scipp.ucsc.edu, zoharko@ias.edu

ABSTRACT: We prove a general bound on the superpotential in theories with broken supersymmetry and broken $R$-symmetry, $|\langle W\rangle|<\frac{1}{2} f_{a} F$, where $f_{a}$ and $F$ are the $R$-axion and Goldstino decay constants, respectively. The bound holds for weakly coupled as well as strongly coupled theories, thereby providing an exact result in theories with broken supersymmetry. We briefly discuss several possible applications.

KEYwords: Supersymmetry Breaking, Supersymmetric Effective Theories

ARXIV EPRINT: 0910.2527 


\section{Contents}

1 Introduction 1

2 The bound in (gauged) O'Raifeartaigh-like models 3

2.1 Proof of the bound for O'Raifeartaigh-like models 3

2.2 Examples 4

2.3 Adding $D$-terms

3 The bound in sigma models $\quad 5$

$\begin{array}{lll}3.1 & \text { An overture to beyond the sigma model } & 6\end{array}$

4 A proof of the bound via low energy effective field theory $\quad 8$

4.1 A review of nonlinear SUSY and $R$-symmetry 9

4.2 General proof of the bound 11

5 Conclusions 13

$\begin{array}{ll}\text { A Low energy analysis of a simple sigma model } & 14\end{array}$

\section{Introduction}

In models with rigid supersymmetry, $R$-symmetry is intimately tied to questions of supersymmetry breaking. The theorem of Nelson and Seiberg [1] asserts that a calculable (and generic) supersymmetric theory exhibits supersymmetry breaking only if it possesses an $R$-symmetry, and that a generic calculable theory with broken $R$-symmetry breaks supersymmetry. Metastable breaking, one expects, will usually require an approximate $R$-symmetry [2].

In a supersymmetric and $R$-symmetric theory, one is not allowed to add an arbitrary constant to the superpotential. It can only be generated dynamically in the IR upon $R$-symmetry breaking. Thus, in $R$-symmetric theories, a candidate order parameter for $R$-symmetry breaking is the superpotential itself. As we will see, in $R$ symmetric theories, $\langle W\rangle$ is indeed a good order parameter, as it is directly measurable in low energy scattering experiments. It is therefore natural to ask what is the relation of the superpotential VEV to other order parameters in the problem, such as the $R$-axion decay constant and the vacuum energy (which is related to the Goldstino decay constant). Understanding $\langle W\rangle$ could elucidate the connection between $R$-symmetry breaking and SUSY breaking [1].

An additional circumstance in which $\langle W\rangle$ takes on physical significance is in coupling a theory to supergravity. There, $\langle W\rangle$ plays an important role in accommodating a small cosmological constant, and is directly related to the value of the gravitino mass and the mass of any would-be $R$-axion. 
The simplest model one might consider is a free theory of a single chiral superfield $Z$ with $R(Z)=2$

$$
W=f Z, \quad K=Z^{\dagger} Z .
$$

Both the boson and the fermion in $Z$ are massless, and there is a moduli space of SUSYbreaking vacua parameterized by the expectation value of the bottom component of $Z$, $\langle z\rangle$. Writing $z=|z| e^{2 i a}$, we see that the $R$-axion decay constant ${ }^{1}$ is $f_{a}=2|\langle z\rangle|$ while $\langle W\rangle=f\langle z\rangle$ and the vacuum energy density is $|f|^{2}$. So,

$$
|\langle W\rangle|=\frac{1}{2} f_{a} F,
$$

where $F$ is the Goldstino decay constant, given by the vacuum energy $F^{2}=|f|^{2}$.

This trivial model is illuminating. In this paper, we will prove an inequality, which holds in any theory with broken supersymmetry and broken $R$-symmetry:

$$
|\langle W\rangle| \leq \frac{1}{2} f_{a} F .
$$

We will also argue that interacting theories always lead to a strict inequality

$$
|\langle W\rangle|<\frac{1}{2} f_{a} F .
$$

We will prove the result in a sequence of situations. First, in section 2, we will consider general O'Raifeataigh-like models, at tree-level. Here the proof is quite simple. We will illustrate the theorem with models that saturate the bound at tree-level, and which do not, and understand the distinction. We will also consider renormalizable theories with gauge interactions, for which $D$-terms may be non-zero, and show that the inequality remains true.

In section 3, we consider linear sigma models, i.e. theories with chiral fields and a general Kähler potential and superpotential. At the level of two derivative terms in the effective action, the theorem is readily proven for these theories as well. However, for a class of theories which saturate the bound, it is necessary to look at higher orders in the derivative expansion. In this case, the proof of the theorem invokes considerations of unitarity along the lines of [3].

In section 4 we address the most general case, which need not be a calculable theory. To this end we use the machinery of non-linear effective Lagrangians, developed recently in [4]. After reviewing the necessary background, we show that the desired inequality translates into an inequality between various parameters in the effective non-linear Lagrangian. Along with standard manipulations in such effective theories, we use the consistency conditions for effective field theories discussed in [3] to prove that non-trivial interacting theories always satisfy (1.4).

A number of technical details are covered in an appendix.

\footnotetext{
${ }^{1}$ We define the $R$-axion decay constant, $f_{a}$, as the coefficient of the kinetic term of the axion

$$
-f_{a}^{2}(\partial a)^{2}
$$
}




\section{The bound in (gauged) O'Raifeartaigh-like models}

In this section we will prove the bound in theories with canonical Kähler potential where both SUSY and the $R$-symmetry are broken. We will start our analysis from the case of O'Raifeartaigh-like models. We then briefly comment on the extension to models with gauge fields.

The general model with canonical Kähler potential and no gauge interactions takes the form

$$
\mathcal{L}=\int d^{2} \theta d^{2} \bar{\theta} \sum_{i} \bar{\Phi}_{i} \Phi_{i}+\int d^{2} \theta W\left(\Phi_{i}\right)+\text { c.c. }
$$

The chiral fields $\Phi_{i}$ have charge $q_{i}$ under the $R$-symmetry. By assumption $W\left(\Phi_{i}\right)$ is a holomorphic function of the $\Phi_{i}$ of $R$-charge 2 . Before proving our inequality in these models, we would like to outline rather general features that are helpful to develop some intuition.

Let us consider theories of the form (2.1) that have a SUSY-breaking and $R$-symmetry breaking vacuum at some point $\phi_{i}^{(0)}$, where $\phi_{i}$ is the bottom component of $\Phi_{i}$. The spectrum therefore comprises a massless Goldstino and a massless $R$-axion. As discussed in [5], classically, there is always a complex massless boson (in fact a whole flat direction) generated by the transformation

$$
\phi_{i}^{(0)} \rightarrow \phi_{i}^{(0)}+\alpha\left(\frac{\partial W}{\partial \phi_{i}}\right)^{*}
$$

for any complex $\alpha$. A simple way to remember this massless direction is as the bosonic superpartner of the Goldstino [6].

One can distinguish two cases:

- If the $R$-symmetry is broken everywhere on the pseudomoduli space (2.2), the Goldstino superpartner is linearly independent of the $R$-axion [6]. As a consequence, generically, the pseudomoduli space is three real dimensional.

- If somewhere on the pseudomoduli space (2.2) the $R$-symmetry is restored then often these are the only two real flat directions. The $R$-axion is embedded into this complex flat direction as some phase coordinate around the $R$-restoring point.

We will see that the first case satisfies the inequality $|\langle W\rangle|<\frac{1}{2} f_{a} F$ while, as in the second case, if the $R$-axion direction is embedded in (2.2) we get $|\langle W\rangle|=\frac{1}{2} f_{a} F$.

\subsection{Proof of the bound for O'Raifeartaigh-like models}

Since, by assumption, $W\left(\Phi_{i}\right)$ is a holomorphic function of the $\Phi_{i}$ of $R$-charge 2 we have the following identity $\left(q_{i}\right.$ stands for the $R$-charge of $\Phi_{i}$ )

$$
2 W\left(\Phi_{i}\right)=\sum_{j} q_{j} \Phi_{j} \frac{\partial W\left(\Phi_{i}\right)}{\partial \Phi_{j}} .
$$


We now consider the bottom component of (2.3) and define two complex vectors $w_{i}=q_{i} \phi_{i}$ and $F_{i}^{\dagger}=\frac{\partial W}{\partial \phi_{i}}$. The Goldstino decay constant $F$ is given by ${ }^{2}$

$$
F^{2}=\sum_{j} F_{j}^{\dagger} F_{j}=\langle\nabla \mathbf{W}, \nabla \mathbf{W}\rangle
$$

The scalar fields are parameterized by the $R$-axion $a$ as $\phi_{i}=\left|\phi_{i}\right| e^{i q_{i} a}$. The kinetic part of the Lagrangian for $a$ is:

$$
-\partial_{\mu} a \partial^{\mu} a \sum_{i} q_{i}^{2}\left|\phi_{i}\right|^{2}
$$

from which we read the $R$-axion decay constant $f_{a}^{2}=\sum_{i} q_{i}^{2}\left|\phi_{i}^{(0)}\right|^{2}=\langle\mathbf{w}, \mathbf{w}\rangle$.

We use the Cauchy-Schwarz inequality in (2.3) and obtain

$$
4|W|^{2}=|\langle\mathbf{F}, \mathbf{w}\rangle|^{2} \leq\langle\mathbf{w}, \mathbf{w}\rangle\langle\nabla \mathbf{W}, \nabla \mathbf{W}\rangle .
$$

This can be rewritten as $2|\langle W\rangle| \leq f_{a} F$, establishing the bound.

An immediate corollary is that we can classify the models which saturate the bound. $R$-symmetry transformations are generated by

$$
\phi_{i}^{(0)} \rightarrow \phi_{i}^{(0)}+i \epsilon q_{i} \phi_{i}^{(0)} .
$$

From (2.6) we see that the bound is saturated if and only if the vector $w_{i}=q_{i} \phi_{i}^{(0)}$ is proportional to $F_{i}=W_{i}^{*}$. This means that the $R$-symmetry transformation (2.7) is part of the canonical pseudomodulus space (2.2). Theories in which the canonical pseudomoduli space (2.2) does not contain an $R$-symmetric point cannot saturate the bound.

\subsection{Examples}

As a first example consider the original O'Raifeartaigh model [7] with superpotential

$$
W=X\left(\frac{\lambda}{2} A^{2}-f\right)-m Y A
$$

where $X$ and $Y$ have $R$-charge 2 while $A$ is neutral. This model is generic under the assumption of an extra $Z_{2}$ symmetry changing the sign of $A$ and $Y$. For $m^{2} \geq \lambda f$ the lowest lying pseudomodulus space of vacua is given by $Y=A=0$. The only field with a nonzero $F$-term is $X$ and $F_{X}^{\dagger}=f$. On this branch $W_{\text {eff }}=-f X$. The $R$-axion is embedded in the phase of $X$ with $f_{a}=2|X|$. Then $f_{a} F=2|X||f|=2|W|$ and the bound is saturated. The story for the other branch (which is stable for $m^{2} \leq \lambda f$ ) of the O'Raifeartaigh model (2.8) is very similar.

A model where the $R$-symmetry is broken everywhere on the pseudomoduli space was given in [8]; this class of models was considered in more generality in [6] and [9]. As an example consider the following superpotential:

$$
W=X\left(\gamma \phi_{2 / 3} \phi_{-2 / 3}-\mu^{2}\right)+\frac{\delta}{3} \phi_{2 / 3}^{2} \chi_{2 / 3}+m_{1} \phi_{2 / 3} Y_{4 / 3}+m_{2} \phi_{-2 / 3} Z_{8 / 3}+\lambda \chi_{2 / 3}^{3} .
$$

\footnotetext{
${ }^{2}$ The inner product $\langle$,$\rangle is the standard (Hermitian) Euclidian inner product.$
} 
The subscripts are the $R$-charges of the various fields. $X$ has $R$-charge 2 . This superpotential is the most general compatible with a $Z_{2}$ symmetry under which all fields but $X$ and $\chi_{2 / 3}$ are odd. When $\sigma$, defined by $\mu^{2}=\frac{m_{1} m_{2}}{\gamma}\left(1+\sigma^{2} \gamma^{2}\right)$, is real there is a stable branch of the pseudomoduli space where the $R$-symmetry is everywhere broken:

$$
\phi_{\frac{2}{3}}=m_{2} \sigma e^{i \theta}, \quad \phi_{-\frac{2}{3}}=m_{1} \sigma e^{-i \theta}, \quad \chi_{\frac{2}{3}}=i m_{2} \frac{1}{3} \sqrt{\frac{\delta}{\lambda}} \sigma e^{i \theta}
$$

while the fields with nonzero $F$-terms are related by:

$$
Z_{\frac{8}{3}}=-\gamma \sigma X e^{i \theta}, \quad Y_{\frac{4}{3}}=Z_{\frac{8}{3}} e^{-2 i \theta}-\frac{2 m_{2}^{2} \delta^{\frac{3}{2}}}{3 m_{1} \lambda^{\frac{1}{2}}} \sigma e^{2 i \theta} .
$$

We see that the pseudomoduli space is three real dimensional, in accord with the fact that the $R$-symmetry is nowhere restored.

On this branch the total vacuum energy (and hence the Goldstino decay constant) is

$$
F^{2}=\frac{m_{1}^{2} m_{2}^{2}}{\gamma^{2}}\left(1+2 \sigma^{2} \gamma^{2}\right) \leq \mu^{4}
$$

The $R$-axion is proportional to $\theta(x)$. It can checked explicitly that $2|W|<f_{a} F$ everywhere on the pseudomoduli space for $\sigma \neq 0$. For $\sigma=0$ the $R$-symmetry is restored at $X=0$ and the bound is saturated. The resulting expressions are cumbersome in general, therefore, we just quote the result at leading order for large $|X|$

$$
f_{a}^{2} F^{2}-4|W|^{2}=\frac{8}{9} m_{1}^{2} m_{2}^{2} \sigma^{2}\left(1+2 \gamma^{2} \sigma^{2}\right)|X|^{2}+O\left(X^{0}\right) \geq 0 .
$$

\section{$2.3 \quad$ Adding $D$-terms}

The discussion above is readily generalized to include possible $D$-terms. Indeed, we can repeat the discussion around eqs. (2.3) and (2.5). The only change is that the Goldstino decay constant, $F$, receives additional contributions,

$$
F^{2}=\sum_{i}\left|\frac{\partial W}{\partial \phi_{i}}\right|^{2}+\sum_{a} D_{a}^{2}
$$

So $\left.F^{2}\right\rangle\langle\nabla \mathbf{W}, \nabla \mathbf{W}\rangle$, strengthening the bound.

\section{The bound in sigma models}

In this section, we show that the bound is satisfied for theories described by arbitrary superpotential and Kähler potential (consistent with an $R$-symmetry)

$$
K\left(\phi_{i}, \bar{\phi}_{j}\right), \quad W\left(\phi_{i}\right)
$$

Let us denote for simplicity

$$
\mathcal{M}_{l \bar{m}}=\frac{\partial^{2} K}{\partial \phi_{l} \partial \bar{\phi}_{m}}
$$


The matrix $g=\mathcal{M}+\mathcal{M}^{\dagger}$ is Hermitian. It is also positive definite around the configurations in field space we are interested in, since otherwise there are ghosts. Therefore, we can decompose

$$
g=L L^{\dagger}, \quad g^{-1}=\left(L^{\dagger}\right)^{-1} L^{-1} .
$$

Even when the Kähler potential is non-canonical, (2.3) still holds and we can rewrite (by inserting the identity matrix ${ }^{3}$ )

$$
2 W=\sum_{i} q_{i} \phi_{i} \frac{\partial W}{\partial \phi_{i}}=\sum_{i, j, k} q_{i} \phi_{i} L_{i j} L_{j k}^{-1} \frac{\partial W}{\partial \phi_{k}} .
$$

It is useful to define the two vectors $w_{j}=\sum_{i} q_{i} \phi_{i} L_{i j}, v_{j}^{\dagger}=\sum_{k} L_{j k}^{-1} \frac{\partial W}{\partial \phi_{k}}$. The Goldstino decay constant, $F$, is related to the vacuum energy which is just $F^{2}=\langle\mathbf{v}, \mathbf{v}\rangle$, while the $R$-axion decay constant can be read off the kinetic terms of the sigma model (3.1). The kinetic terms contain the matrix $g$ and therefore the decay constant satisfies $f_{a}^{2}=\langle\mathbf{w}, \mathbf{w}\rangle$.

Using the Cauchy-Schwarz inequality again we get

$$
4|W|^{2}=|\langle\mathbf{v}, \mathbf{w}\rangle|^{2} \leq\langle\mathbf{w}, \mathbf{w}\rangle\langle\mathbf{v}, \mathbf{v}\rangle=f_{a}^{2} F^{2},
$$

or in other words

$$
2|W| \leq f_{a} F
$$

which confirms the bound we claim.

\subsection{An overture to beyond the sigma model}

While very suggestive, however, the tree-level argument in the general sigma model does not prove the theorem in complete generality. There are two limitations. First, while many models of dynamical supersymmetry breaking (in particular models where SUSY is broken at tree-level) permit a low energy description of SUSY breaking in terms of a field theory with linearly realized supersymmetry, there are many models which do not. Perhaps the most well known examples are the SU(5) and SO(10) models of [10, 11]. Secondly, we have only considered tree-level sigma models, forbidding derivative corrections in the Kähler potential and (not dissimilar) radiative corrections.

To show that derivative corrections in the Kähler potential may play a decisive role, consider the following tree-level theory of a single chiral superfield $Z$ with $R$-charge 2

$$
K=K\left(Z Z^{\dagger}\right), \quad W=f Z .
$$

Such models arise as an effective description of theories with a characteristic mass scale $M$ and a parameterically small SUSY breaking scale $|f| \ll M^{2}$. This separation of scales guarantees that one should be able to describe the theory at low energies in terms of an effective action with linearly realized supersymmetry.

Repeating our analysis above in this simple case, one sees that the bound is saturated (since the $R$-symmetry breaking must occur, if at all, in the same direction as SUSY

\footnotetext{
${ }^{3}$ Note that the index $j$ takes values in both barred and unbarred indices, while $i, k$ only take values in the unbarred indices. We hope our attempt not to clutter the notation will not cause a confusion.
} 
breaking). The description (3.7) is a valid effective description up to two derivatives. But generically, there are also higher derivative corrections.

At the level of terms with two derivatives in superspace, the effective action may include various contributions. Let us study, for example, the term

$$
-\int d^{4} \theta \frac{\epsilon}{M^{4}} Z^{\dagger} Z \partial_{\mu} Z^{\dagger} \partial^{\mu} Z \text {. }
$$

This term changes $f_{a}$ without affecting the Goldstino decay constant. Indeed since it has explicit derivatives it has no effect on the vacuum energy, but it does change the normalization of the kinetic term for $z$. In addition, this term does not change the superpotential $\operatorname{VEV}\langle W\rangle{ }^{4}$

Since the original theory (3.7) always saturates the bound, a negative sign for $\epsilon$ would seem to contradict the inequality: the change in $f_{a}$ in the presence of (3.8) is such that $f_{a}$ decreases if $\epsilon$ is negative. Therefore, some principle should dictate that $\epsilon$ is positive if the inequality is true.

This is the first point where we see the importance of unitarity arguments in effective field theory. The operator (3.8) can be shown to arise with a definite sign of $\epsilon$ in effective theories that have UV completions. In the rest of this subsection we will show that this is the case by carefully studying the theory (3.7) deformed by (3.8).

We define the $R$-axion as usual to be the phase of $z, z=|z| e^{2 i a}$. Suppose for a moment that $\epsilon=0$. The Lagrangian, after integrating out the radial mode $|z|$, contains the usual kinetic terms for the axion and Goldstino, but it also contains some interaction terms between the axion and the Goldstino. As we show in appendix $\aleph$, the leading interaction is of the form

$$
\mathcal{L}_{\text {interaction }} \approx c_{1} \psi^{2} \partial_{\mu} a \partial^{\mu} a+\text { c.c. } .
$$

Another possible coupling one could imagine has three derivatives and takes the form $i\left(\partial^{\nu} \psi \sigma^{\mu} \bar{\psi}\right) \partial_{\mu} a \partial_{\nu} a$, however, as we show explicitly in appendix $\aleph$, it does not arise at tree-level in the theory with $\epsilon=0$.

Introducing the deformation (3.8) induces this new coupling of two fermions to two axions, with coefficient proportional to $\epsilon$. We will now argue that, very similarly to examples discussed in [3], the theory

$$
\mathcal{L}=-f_{a}^{2}\left(\partial_{\mu} a\right)^{2}+i \partial_{\mu} \bar{\psi} \bar{\sigma}^{\mu} \psi+\left(c_{1} \psi^{2}\left(\partial_{\mu} a\right)^{2}+\text { c.c. }\right)-i c_{2}\left(\partial^{\nu} \psi \sigma^{\mu} \bar{\psi}\right) \partial_{\mu} a \partial_{\nu} a \cdots,
$$

where $\cdots$ stand for self interactions of the axion and the Goldstino that we are not interested in, has superluminal modes if $c_{2}$ has the wrong sign.

Let us look at the propagation of the Goldstino $\psi$ in a background with $\partial_{\mu} a=V_{\mu}$, where $V_{\mu}$ is a constant four vector. The Lagrangian for the Goldstino becomes

$$
\mathcal{L}=i \partial_{\mu} \bar{\psi} \bar{\sigma}^{\mu} \psi+\left(c_{1} V^{2} \psi^{2}+\text { c.c. }\right)-i c_{2} V_{\mu} V_{\nu} \partial^{\nu} \psi \sigma^{\mu} \bar{\psi}+\cdots
$$

We are expanding in small $V$, as appropriate in effective field theory. Note that around the background $\partial_{\mu} a=V_{\mu}$ the fermion $\psi$ is massive with mass $\sim V^{2}$. As usual, in the final

\footnotetext{
${ }^{4}$ In the next section we will define the observable $\langle W\rangle$ more carefully.
} 
dispersion relation the mass appears squared, so it can be dropped. The remaining terms yield the following equation of motion in momentum space

$$
\left(k_{\mu} \bar{\sigma}^{\mu}-c_{2}(V \cdot k) V_{\mu} \bar{\sigma}^{\mu}\right) \psi=0 .
$$

Let us multiply this equation by $V_{\rho} \sigma^{\rho}$ from the left. We see that at leading order in $V$

$$
V_{\rho} k_{\mu} \sigma^{\rho} \bar{\sigma}^{\mu} \psi=0 .
$$

The next step is to multiply (3.12) by $k_{\rho} \sigma^{\rho}$ from the left to obtain

$$
\left(-k^{2} \delta_{\beta}^{\alpha}-c_{2}(V \cdot k) k_{\rho} V_{\mu}\left(\sigma^{\rho} \bar{\sigma}^{\mu}\right)_{\beta}^{\alpha}\right) \psi_{\alpha}=0 .
$$

We can symmetrize the second term using (3.13). We obtain

$$
\left(k^{2}-2 c_{2}(V \cdot k)^{2}\right) \psi_{\beta}=0 .
$$

We see that the dispersion relation is corrected by $(V \cdot k)^{2}$, which has a definite sign. This leads to superluminal modes unless $c_{2} \geq 0$.

Expanding the term (3.8) in terms of component fields, we find that it leads to an operator of the form $c_{2}$ with coefficient $c_{2}=8 \epsilon v^{2} / M^{4}$, where $v$ is the VEV of $|z|$. If the operator of eq. (3.8) were the only four derivative operator, we see that unitarity would require the coefficient $\epsilon$ to be positive. ${ }^{5}$ As we commented above, a positive $\epsilon$ increases the decay constant of the $R$-axion, leaving the other quantities intact, therefore, we would find, for any non-zero $\epsilon$,

$$
2|W|<f_{a} F .
$$

The operator of eq. (3.8) is just one of several operators which can potentially correct $f_{a}$. In order to provide a general proof of the bound, we will see that it is necessary to consider the effective theory at very low energies.

In spite of the fact that at tree-level the bound can sometimes be saturated, we will show that nontrivial interacting theories will always satisfy (3.16). It is not difficult to verify that in simple perturbative models, such as those of [12], the bound is indeed satisfied at one-loop.

\section{A proof of the bound via low energy effective field theory}

In the previous two sections we have seen how the bound arises in tractable field theories and we have also witnessed the importance of unitarity arguments in effective field theory. The arguments of the previous sections cover a variety of models, but not the interesting, and potentially important ones where the scale of supersymmetry breaking is not small compared to other characteristic mass scales of the theory, so that SUSY breaking cannot be described by a superpotential with linearly realized SUSY. Models of the latter kind are

\footnotetext{
${ }^{5}$ The connection between superluminal modes and unitarity bounds was demonstrated in [3]. These ideas apply in our context equally well. In particular, while the absence of superluminal modes only suggests that $c_{2} \geq 0$, unitarity gives $c_{2}>0$ because it relates $c_{2}$ to an integral of a total cross section.
} 
pervasive; examples include the $\mathrm{SU}(5)$ and $\mathrm{SO}(10)$ models of [10, 11]. In addition, while in the previous section we discussed one particular derivative correction characteristic of perturbative models, we would like to be able to control all of them along with all the possible radiative corrections.

Therefore, our goal in this section is to extend the validity of our bound to these cases as well. All we know is that these models break supersymmetry and have a spontaneously broken $R$-symmetry. Therefore, the low energy spectrum consists of an $R$-axion and a Goldstino. However, this cannot be an arbitrary effective theory, rather, it has to be constrained by nonlinearly realized SUSY and $R$-symmetry.

As explained in [4], such nonlinear theories are most conveniently organized in terms of a set of superfields that satisfy algebraic constraints. Below we review this formalism, focusing on the aspects pertinent to this work. After reviewing the necessary ingredients, we will derive the bound

$$
2|W|<f_{a} F,
$$

establishing its validity as a nonperturbative result.

\subsection{A review of nonlinear SUSY and $R$-symmetry}

The low energy effective theory we are after includes the Goldstino $G_{\alpha}(x)$ and the $R$ axion $a(x)$. The Lagrangian should respect nonlinearly realized supersymmetry as well as nonlinearly realized $R$-symmetry. The latter is easier to understand, as it includes an inhomogeneous shift of the $R$-axion accompanied by a rotation of the Goldstino field and the superspace coordinate

$$
a \rightarrow a+\xi, \quad G_{\alpha} \rightarrow e^{i \xi} G_{\alpha}, \quad \theta_{\alpha} \rightarrow e^{i \xi} \theta_{\alpha} .
$$

On the other hand, the action of nonlinear supersymmetry is more complicated since the underlying group structure is non-Abelian. There are many approaches to nonlinear realizations of supersymmetry, see e.g. [13] (more references can be found in [4]). In this work we will adopt the conventions and approach of [4] as it easily allows to describe offshell effective actions that may or may not include particles in addition to the Goldstino. ${ }^{6}$

The main point is that we will use the power of supersymmetry and superfields, but our superfields will satisfy some constraints. The effect of these constraints, as we will momentarily see, is to remove some degrees of freedom from the conventional superfields.

To see how this works, we start from a chiral superfield, $X_{\mathrm{NL}}$, that satisfies the constraint

$$
X_{\mathrm{NL}}^{2}=0
$$

This constraint eliminates the complex boson in the bottom component of $X_{\mathrm{NL}}$ but leaves the fermion component as well as an auxiliary field. The solution to (4.3) is

$$
X_{\mathrm{NL}}=\frac{G^{2}}{2 F_{X}}+\sqrt{2} \theta G+\theta^{2} F_{X}
$$

\footnotetext{
${ }^{6}$ Some comments on the relation between [4] and previous approaches can be found in [14].
} 
where all the variables are functions of $y^{\mu}=x^{\mu}+i \theta \sigma^{\mu} \bar{\theta}$. It is therefore natural to identify the fermion surviving the constraint (4.3) as the Goldstino.

If the Goldstino is the only massless particle, we can easily write effective Lagrangians by using superspace and the superfield $X_{\mathrm{NL}}$. These by construction respect nonlinear SUSY. The simplest possible Lagrangian is

$$
\mathcal{L}=\int d^{4} \theta X_{\mathrm{NL}}^{\dagger} X_{\mathrm{NL}}+\left(\int d^{2} \theta f X_{\mathrm{NL}}+\text { c.c. }\right) .
$$

This is no other than the Akulov-Volkov theory [15], that in components includes the following terms. ${ }^{7}$

$$
\mathcal{L}=-f^{2}+i \partial_{\mu} \bar{G} \bar{\sigma}^{\mu} G+\frac{1}{4 f^{2}} \bar{G}^{2} \square G^{2}+\cdots
$$

where ... stand for terms with more Goldstinos.

While the constraint (4.3) forbids a nontrivial Kähler potential, corrections to (4.5) with derivatives are allowed (and generally appear from microscopic models). The natural way to control these corrections is to assign the Goldstino $G_{\alpha}$ effective scaling dimension $-1 / 2$ and therefore the superfield $X_{\mathrm{NL}}$ has scaling dimension -1

$$
S(X)=-1
$$

This choice forces us to assign $d \theta$ scaling dimension $+1 / 2$. The terms in the Lagrangian (4.5) then have scaling dimension zero. It can be proven [4] that at scaling zero the Lagrangian (4.5) is the most general possible up to field redefinitions. The theory (4.5) has only one free parameter, the SUSY breaking scale.

To include an $R$-axion (or more generally a Goldstone boson) we introduce a chiral superfield $\mathcal{A}_{\mathrm{NL}}$, satisfying the following constraint

$$
X_{\mathrm{NL}}\left(\mathcal{A}_{\mathrm{NL}}-\mathcal{A}_{\mathrm{NL}}^{\dagger}\right)=0 .
$$

Out of all the degrees of freedom in a conventional chiral superfield, the constraint (4.8) leaves only one real degree of freedom (and no auxiliary fields). In components, the superfield $\mathcal{A}_{\mathrm{NL}}$ takes the form

$$
\mathcal{A}_{\mathrm{NL}}=H+i \sqrt{2} \theta \sigma^{\mu}\left(\frac{\bar{G}}{\bar{F}_{X}}\right) \partial_{\mu} H+\theta^{2}\left(-\partial_{\nu}\left(\frac{\bar{G}}{\bar{F}_{X}}\right) \bar{\sigma}^{\mu} \sigma^{\nu} \frac{\bar{G}}{\bar{F}_{X}} \partial_{\mu} H+\frac{1}{2 \bar{F}_{X}^{2}} \bar{G}^{2} \square H\right),
$$

where

$$
H=a+\frac{i}{2}\left(\frac{G}{F_{X}} \sigma^{\mu} \frac{\bar{G}}{\bar{F}_{X}}\right) \partial_{\mu} a+\cdots
$$

where the ellipses stand for terms with more fermions and derivatives. We will not need them here. ${ }^{8}$

We see that $R$-symmetry acts on $\mathcal{A}_{\mathrm{NL}}$ by shifts $\mathcal{A}_{\mathrm{NL}} \rightarrow \mathcal{A}_{\mathrm{NL}}+\xi$. Of course, the shift preserves the constraint (4.8). Due to this action of $R$-symmetry it is natural to define an exponentiated superfield

$$
\mathcal{R}_{\mathrm{NL}}=e^{i \mathcal{A}_{\mathrm{NL}}}
$$

\footnotetext{
${ }^{7}$ For alternative descriptions of this theory see [16-20].

${ }^{8}$ The corrections are easily derived from (4.8). An explicit expression is given in [4].
} 
which under $R$-symmetry transforms as $\mathcal{R}_{\mathrm{NL}} \rightarrow e^{i \xi} \mathcal{R}_{\mathrm{NL}}$. The constraint (4.8) becomes

$$
X_{\mathrm{NL}}\left(\mathcal{R}_{\mathrm{NL}}^{\dagger} \mathcal{R}_{\mathrm{NL}}-1\right)=0
$$

We are now ready to write Lagrangians. As we are going to use superspace and superfields, both nonlinear supersymmetry and nonlinear $R$-symmetry will be manifest. Following the same idea as around (4.5) we get the following Lagrangian

$$
\mathcal{L}_{\text {Goldstino-axion }}=\int d^{4} \theta\left(\left|X_{\mathrm{NL}}\right|^{2}+f_{a}^{2}\left|\mathcal{R}_{\mathrm{NL}}\right|^{2}\right)+\int d^{2} \theta\left(f X_{\mathrm{NL}}+\widetilde{f} \mathcal{R}_{\mathrm{NL}}^{2}\right)+\text { c.c. } .
$$

The natural scaling dimension of the $R$-axion is zero and so

$$
S\left(\mathcal{R}_{\mathrm{NL}}\right)=0
$$

We see that the effective Lagrangian contains three independent parameters. $f$ and $f_{a}$ are identified as the SUSY breaking scale and the $R$-axion decay constant, respectively. The remaining parameter $\widetilde{f}$ corresponds to the VEV of the superpotential.

Similarly to the case in (4.5), it can be shown that in an expansion in derivatives (more precisely, in the scaling $S$ ) the theory (4.13) is the leading universal theory at low energies (up to field redefinitions that can be absorbed in redefinitions of $f, f_{a}, \widetilde{f}$ ).

Let us pause for a moment to discuss the parameter $\tilde{f}$. In theories that break supersymmetry, the VEV of the superpotential is, in general, not a holomorphic function of the superpotential couplings. Furthermore, in the presence of covariant derivatives its naive definition is ambiguous. ${ }^{9}$ The parameter $\tilde{f}$ provides a precise physical definition of $\langle W\rangle$, which generalizes what one would naively call the VEV of the superpotential in simple theories like those analyzed in the previous sections.

We conclude that if we wish to analyze the interactions between an $R$-axion and the Goldstino at very low energies, we should study the theory (4.13) and substitute the component expressions (4.4), (4.9), (4.10) for the superfields $X_{\mathrm{NL}}, \mathcal{R}_{\mathrm{NL}}$. This is done in detail in the next subsection, where we provide the most general proof of the bound.

\subsection{General proof of the bound}

In this section we will describe the interactions resulting from (4.13). We will only keep terms with at most four fields, as this will suffice for the argument we are about to make. We directly substitute the expressions (4.4), (4.9), (4.10) into (4.13) and get the following

$$
\begin{aligned}
\mathcal{L}_{\text {Goldstino-axion }}= & -f^{2}+i \partial_{\mu} \bar{G} \bar{\sigma}^{\mu} G-f_{a}^{2}(\partial a)^{2}+\frac{1}{4 f^{2}} \bar{G}^{2} \square G^{2}-2 i \frac{f_{a}^{2}}{f^{2}} \partial^{\mu} G \sigma^{\lambda} \bar{G} \partial_{\mu} a \partial_{\lambda} a \\
& +\left(\frac{\tilde{f}}{f^{2}} \bar{G} \bar{\sigma}^{\mu} \sigma^{\nu} \partial_{\nu} \bar{G} \partial_{\mu}\left(e^{2 i a}\right)-\frac{i \widetilde{f}}{f^{2}} \bar{G}^{2} e^{2 i a} \square a+\frac{2 \widetilde{f}}{f^{2}} \bar{G}^{2}(\partial a)^{2}+\text { c.c. }\right) .
\end{aligned}
$$

We have dropped quartic terms that are proportional to the free equations of motion, as they effectively contain more than four fields. Note that the first two terms in the

\footnotetext{
${ }^{9}$ For example, because we can always add to the superpotential terms like $\bar{D}^{2} \mathcal{O}^{\dagger}$ with $\mathcal{O}^{\dagger}$ anti-chiral and $R$-neutral. This does not change the physical theory but may affect the value of $\langle W\rangle$.
} 
second line are proportional to the free equations of motion of the Goldstino and the axion, respectively. However, the key point is that these are cubic terms, so they can lead to effective quartic operators.

To calculate the quartic terms we may use field redefinitions to eliminate the cubic terms. Let us define a new Goldstino field $\widetilde{G}_{\alpha}$ and a new axion $\widetilde{a}$ related to the variables in (4.15) via

$$
\widetilde{G}=G+i \frac{\widetilde{f}}{f^{2}} \bar{G} \bar{\sigma}^{\mu} \partial_{\mu}\left(e^{2 i a}\right), \quad \widetilde{a}=a-i \frac{\widetilde{f}}{2 f^{2} f_{a}^{2}} e^{2 i a} \widetilde{\widetilde{G}}^{2}+c . c .
$$

Note that these new Goldstino and axion fields are not the same as those sitting in the superfields, $X_{\mathrm{NL}}, \mathcal{R}_{\mathrm{NL}}$. For example, their transformation laws under supersymmetry are different. The Lagrangian (4.15) can be written in terms of the new fields (4.16). The result (again, up to terms with more than four fields) is

$$
\begin{aligned}
\mathcal{L}_{\text {Goldstino-axion }}= & -f^{2}+i \partial_{\mu} \overline{\widetilde{G}} \bar{\sigma}^{\mu} \widetilde{G}-f_{a}^{2}(\partial a)^{2}+\left(\frac{1}{4 f^{2}}-\frac{|\widetilde{f}|^{2}}{2 f^{4} f_{a}^{2}}\right) \widetilde{\widetilde{G}}^{2} \square \widetilde{G}^{2} \\
& -2 i\left(\frac{f_{a}^{2}}{f^{2}}-4 \frac{|\widetilde{f}|^{2}}{f^{4}}\right) \partial^{\mu} \widetilde{G} \sigma^{\lambda} \overline{\widetilde{G}} \partial_{\mu} a \partial_{\lambda} a \\
& +\left(\frac{2 \widetilde{f}}{f^{2}} \widetilde{G}^{2}(\partial a)^{2}+\frac{\left(\widetilde{f}^{*}\right)^{2}}{4 f^{4} f_{a}^{2}} \widetilde{G}^{2} \square \widetilde{G}^{2}+\text { c.c. }\right) .
\end{aligned}
$$

Let us now analyze the theory (4.17). The most interesting term is the first operator in the second line of $(4.17),-i \partial^{\mu} \widetilde{G} \sigma^{\lambda} \overline{\widetilde{G}} \partial_{\mu} a \partial_{\lambda} a$. In section 3 , we have shown that the coefficient must be non-negative to avoid superluminal propagation. Unitarity provides a slightly stronger constraint. As in [3], the coefficient of the operator $-i \partial^{\mu} \widetilde{G} \sigma^{\lambda} \widetilde{\widetilde{G}} \partial_{\mu} a \partial_{\lambda} a$ can be related to an integral of a total cross section, therefore once we include radiative corrections, we expect the coefficient to be strictly positive. This means that

$$
\frac{f_{a}^{2}}{f^{2}}-4 \frac{|\widetilde{f}|^{2}}{f^{4}}>0
$$

or equivalently,

$$
2|\widetilde{f}|<f_{a} f .
$$

Since $\widetilde{f}$ is just the VEV of the superpotential we conclude with the claimed bound

$$
2|W|<f_{a} F,
$$

where $F$, as always, is the Goldstino decay constant (which is identical to the total vacuum energy).

As long as (4.19) is satisfied the coefficient of the operator $\overline{\widetilde{G}}^{2} \square \widetilde{G}^{2}$ is positive (and in fact bounded from below by $\frac{1}{8 f^{2}}$ ). This is important as one can show that unitarity demands the coefficient of $\widetilde{\widetilde{G}}^{2} \square \widetilde{G}^{2}$ to be positive. ${ }^{10}$

\footnotetext{
${ }^{10}$ Similar unitarity constraints on fermionic vertices were considered in [21].
} 
A simple consistency check on (4.17) is to contrast it with the explicit model in appendix $\aleph$ which at tree-level satisfies $2|W|=f_{a} F$. (Of course, quantum corrections will turn it to a strict inequality.) By plugging into (4.17) $2 \widetilde{f}=f_{a} f$ we recover precisely the low energy effective action we computed explicitly in the appendix by integrating out heavy particles.

\section{Conclusions}

In this paper we have demonstrated an exact result in theories that break supersymmetry. We have used the methods of spontaneously broken symmetries along with unitarity bounds. This has led to a bound involving the VEV of the superpotential, the $R$-axion decay constant and the SUSY breaking scale

$$
|\langle W\rangle|<\frac{1}{2} f_{a} F .
$$

This holds in strongly coupled models in which we do not even know the appropriate variables to describe SUSY breaking macroscopically. A simple (and somewhat surprising at first sight) corollary of (5.1) is that when SUSY is unbroken, even if $R$-symmetry is broken, a nonzero VEV for the superpotential cannot be generated.

While it is satisfying, in and of itself, that one can prove exact results in theories which spontaneously break supersymmetry, we would also like to mention a few possible applications and open questions. The VEV of the superpotential plays a role in supergravity, where it is relevant both for the $R$-axion mass [22] and the cosmological constant. Recently, in [23], it was noted that in theories with discrete $R$-symmetries, the requirement of small cosmological constant constrains their breaking. Indeed, in that reference it was noted that discrete $R$-symmetries often lead to approximate, continuous symmetries, and it was conjectured that the superpotential is bounded roughly along the lines we have established here. As a result, the potential importance of such symmetries depends on the scale $F$.

In addition, since the bound involves quantities relating $R$-symmetry breaking and SUSY breaking, it is feasible that it may lead to a better understanding of the connection between $R$-symmetry breaking and SUSY breaking [1]. We have a great deal of evidence that the observations by Nelson and Seiberg are correct even beyond the regime of validity of their analysis. It would be nice to make this more precise.

It would be satisfying to derive the bound we proved in the language of current algebra. This may lead to further exact results in SUSY-breaking theories. Needless to say, understanding additional general features of supersymmetry-breaking theories is important.

\section{Acknowledgments}

We would like to thank N. Seiberg, D. Shih and C. Marcantonini for interesting discussions. We are grateful to T. Dumitrescu and A. Katz for helpful comments on the manuscript. The work of MD and GF was supported in part by the U.S. Department of Energy. The work of ZK was supported in part by NSF grant PHY-0503584. Any opinions, findings, and conclusions or recommendations expressed in this material are those of the author(s) and do not necessarily reflect the views of the funding agencies. 


\section{A Low energy analysis of a simple sigma model}

This appendix is dedicated to the analysis of the model

$$
K=K\left(Z Z^{*}\right), \quad W=f Z
$$

at low energies. We restrict ourselves to the tree approximation. The simple theory (A.1) enjoys $R$-symmetry under which $R(Z)=2$. We assume that the Kähler potential is such that there exists a SUSY-breaking vacuum at $|z|=v$ where $v$ is nonzero. Therefore, $R$ symmetry is spontaneously broken. The spectrum of the theory thus consists of a massless Goldstino, a massless $R$-axion and a massive real degree of freedom which we, for simplicity, dub the "Higgs field."

We denote the Kähler metric $g_{z z^{*}}=\partial_{Z} \partial_{Z^{*}} K$ and the Christoffel symbols $\Gamma_{z z}^{z}=\frac{g_{z z^{*}, z}}{g_{z z^{*}}}$, $\Gamma_{z^{*} z^{*}}^{z^{*}}=\frac{g_{z z^{*}, z^{*}}}{g_{z z^{*}}}$. The Lagrangian corresponding to (A.1) is given by

$$
\mathcal{L}=-g_{z z^{*}} \partial_{\mu} Z \partial^{\mu} Z^{*}-i g_{z z^{*}} \bar{\psi} \bar{\sigma}^{\mu} D_{\mu} \psi+\left(\frac{f}{2} \Gamma_{z z}^{z} \psi^{2}+\text { c.c. }\right)+\frac{1}{4} R_{z z^{*} z z^{*}} \psi^{2} \bar{\psi}^{2}-\frac{|f|^{2}}{g_{z z^{*}}} .
$$

Here $D_{\mu} \psi=\partial_{\mu} \psi+\Gamma_{z z}^{z}\left(\partial_{\mu} Z\right) \psi$. Since the Kähler potential is only a function of $Z Z^{*}$, so is the metric $g_{z z^{*}}$. By assumption there is a vacuum at $|z|=v$ so we substitute $z=(v+h) e^{2 i a}$ and we see that $g_{z z^{*}}$ is independent of $a$. In addition, the existence of a vacuum for $h=0$ implies that in an expansion around the vacuum

$$
g_{z z^{*}}=\alpha-\frac{\beta}{v^{2}} h^{2}+\cdots
$$

where $\alpha, \beta$ are dimensionless numbers that can be easily determined given a specific model. From this we can also read out the expansion of the Christoffel symbols and curvature around the vacuum

$$
\Gamma_{z z}^{z}=-\frac{\beta}{\alpha v^{2}} h e^{-2 i a}+\cdots, \quad \Gamma_{z^{*} z^{*}}^{z^{*}}=-\frac{\beta}{\alpha v^{2}} h e^{2 i a}+\cdots, \quad R_{z z^{*} z z^{*}}=-\frac{\beta}{2 v^{2}}+\cdots .
$$

We recall that the Higgs field $h$ is massive, and in order to understand the couplings in the IR we should integrate it out. The first approximation is to set it to zero, but we want to read out the low energy effective action more carefully, so we solve the equations of motion of the theory (A.2) at the leading nontrivial order in the number of Goldstinos, axions and derivatives. The full Lagrangian (A.2) truncated to contain only the leading order terms in the Higgs field, according to (A.3), (A.4) is

$$
\begin{aligned}
-\mathcal{L}= & \frac{|f|^{2}}{\alpha}+\alpha(\partial h)^{2}+4 \alpha v^{2}(\partial a)^{2}+i \alpha \bar{\psi} \bar{\sigma}^{\mu} \partial_{\mu} \psi+8 \alpha v h(\partial a)^{2}+\frac{2 \beta}{v} h \partial_{\mu} a \bar{\psi} \bar{\sigma}^{\mu} \psi \\
& +\left(\frac{f \beta}{2 \alpha v^{2}} h e^{-2 i a} \psi^{2}+\text { c.c. }\right)+\frac{\beta}{8 v^{2}} \psi^{2} \bar{\psi}^{2}+\frac{|f|^{2} \beta}{\alpha^{2} v^{2}} h^{2} .
\end{aligned}
$$

The resulting equation of motion for the heavy Higgs field is solved by

$$
h=-\left(\frac{\alpha f}{4|f|^{2}} e^{-2 i a} \psi^{2}+\text { c.c. }\right)-\frac{\alpha^{2} v}{|f|^{2}} \partial_{\mu} a \bar{\psi} \bar{\sigma}^{\mu} \psi-\frac{4 \alpha^{3} v^{3}}{\beta|f|^{2}}(\partial a)^{2} .
$$


We can now plug it back to the action to read out the effective Lagrangian for the axion and Goldstino. We only keep the leading terms describing self interactions of the axion and Goldstino as well as the leading operator connecting the axion with the Goldstino. The final result is

$$
\begin{aligned}
\mathcal{L}_{\text {eff }}= & -\frac{|f|^{2}}{\alpha}-4 \alpha v^{2}(\partial a)^{2}-i \alpha \bar{\psi} \bar{\sigma}^{\mu} \partial_{\mu} \psi+\alpha\left(\frac{\alpha}{4 f^{*}} \psi^{2}+\text { c.c. }\right) \partial^{2}\left(\frac{\alpha}{4 f^{*}} \psi^{2}+\text { c.c. }\right) \\
& +\frac{16 \alpha^{4} v^{4}}{\beta|f|^{2}}(\partial a)^{4}+\left(\frac{2 v \alpha^{2}}{f^{*}} \psi^{2}(\partial a)^{2}+\text { c.c. }\right) .
\end{aligned}
$$

In addition to the expected kinetic terms and vacuum energy, we wrote down the leading interactions of the Goldstino with itself, the axion with itself and the leading operator that connects them. Note that the self interaction of the axion has a positive sign, which is guaranteed by unitarity [3]. A similar comment holds for the term $\psi^{2} \partial^{2}\left(\bar{\psi}^{2}\right)$ which appears in the self interaction of the Goldstino.

The Lagrangian (A.7) takes a more natural form once the Goldstino is canonically normalized (in spite of the change of variables we retain the notation) and we express everything in terms of the decay constant $f_{a}^{2}=4 \alpha v^{2}$ and SUSY breaking scale $F=|f| / \sqrt{\alpha}$. Without loss of generality, we also assume that $f$ is real. We get

$$
\begin{aligned}
\mathcal{L}_{\mathrm{eff}}= & -F^{2}-f_{a}^{2}(\partial a)^{2}-i \bar{\psi} \bar{\sigma}^{\mu} \partial_{\mu} \psi+\left(\frac{1}{4 F} \psi^{2}+\text { c.c. }\right) \partial^{2}\left(\frac{1}{4 F} \psi^{2}+\text { c.c. }\right) \\
& +\frac{\alpha f_{a}^{4}}{\beta F^{2}}(\partial a)^{4}+\left(\frac{f_{a}}{F} \psi^{2}(\partial a)^{2}+\text { c.c. }\right) .
\end{aligned}
$$

We see that two of the interaction terms depend only on $F, f_{a}$ which suggests that they are associated to universal terms in the low energy effective action. On the other hand, the axion quartic interaction depends on the details of the high energy physics (e.g. the parameter $\beta$ ). We show in section 4 that these facts follow from studying nonlinear realizations of broken $R$-symmetry and supersymmetry.

Open Access. This article is distributed under the terms of the Creative Commons Attribution Noncommercial License which permits any noncommercial use, distribution, and reproduction in any medium, provided the original author(s) and source are credited.

\section{References}

[1] A.E. Nelson and N. Seiberg, $R$ symmetry breaking versus supersymmetry breaking, Nucl. Phys. B 416 (1994) 46 [hep-ph/9309299] [SPIRES].

[2] K.A. Intriligator, N. Seiberg and D. Shih, Supersymmetry breaking, R-symmetry breaking and metastable vacua, JHEP 07 (2007) 017 [hep-th/0703281] [SPIRES].

[3] A. Adams, N. Arkani-Hamed, S. Dubovsky, A. Nicolis and R. Rattazzi, Causality, analyticity and an IR obstruction to UV completion, JHEP 10 (2006) 014 [hep-th/0602178] [SPIRES].

[4] Z. Komargodski and N. Seiberg, From linear SUSY to constrained superfields, JHEP 09 (2009) 066 [arXiv:0907.2441] [SPIRES]. 
[5] S. Ray, Some properties of meta-stable supersymmetry-breaking vacua in Wess-Zumino models, Phys. Lett. B 642 (2006) 137 [hep-th/0607172] [SPIRES].

[6] Z. Komargodski and D. Shih, Notes on SUSY and R-symmetry breaking in Wess-Zumino models, JHEP 04 (2009) 093 [arXiv: 0902.0030] [SPIRES].

[7] L. O'Raifeartaigh, Spontaneous symmetry breaking for chiral scalar superfields, Nucl. Phys. B 96 (1975) 331 [SPIRES].

[8] L.M. Carpenter, M. Dine, G. Festuccia and J.D. Mason, Implementing general gauge mediation, Phys. Rev. D 79 (2009) 035002 [arXiv:0805.2944] [SPIRES].

[9] Z. Sun, Tree level spontaneous R-symmetry breaking in O'Raifeartaigh models, JHEP 01 (2009) 002 [arXiv:0810.0477] [SPIRES].

[10] I. Affleck, M. Dine and N. Seiberg, Dynamical supersymmetry breaking in chiral theories, Phys. Lett. B 137 (1984) 187 [SPIRES].

[11] I. Affleck, M. Dine and N. Seiberg, Exponential hierarchy from dynamical supersymmetry breaking, Phys. Lett. B 140 (1984) 59 [SPIRES].

[12] D. Shih, Spontaneous R-symmetry breaking in O'Raifeartaigh models, JHEP 02 (2008) 091 [hep-th/0703196] [SPIRES].

[13] S. Samuel and J. Wess, A superfield formulation of the nonlinear realization of supersymmetry and its coupling to supergravity, Nucl. Phys. B 221 (1983) 153 [SPIRES].

[14] H. Lüo, M. Luo and S. Zheng, Constrained superfields and standard realization of nonlinear supersymmetry, JHEP 01 (2010) 043 [arXiv: 0910.2110] [SPIRES].

[15] D.V. Volkov and V.P. Akulov, Is the neutrino a Goldstone particle?, Phys. Lett. B 46 (1973) 109 [SPIRES].

[16] E.A. Ivanov and A.A. Kapustnikov, Relation between linear and nonlinear realizations of supersymmetry, JINR-E2-10765 [SPIRES].

[17] E.A. Ivanov and A.A. Kapustnikov, General relationship between linear and nonlinear realizations of supersymmetry (in Russian), J. Phys. A 11 (1978) 2375 [SPIRES].

[18] M. Roček, Linearizing the Volkov-Akulov model, Phys. Rev. Lett. 41 (1978) 451 [SPIRES].

[19] U. Lindström and M. Roček, Constrained local superfields, Phys. Rev. D 19 (1979) 2300 [SPIRES].

[20] E.A. Ivanov and A.A. Kapustnikov, The nonlinear realization structure of models with spontaneously broken supersymmetry, J. Phys. G 8 (1982) 167 [SPIRES].

[21] A. Adams, A. Jenkins and D. O'Connell, Signs of analyticity in fermion scattering, arXiv:0802.4081 [SPIRES].

[22] J. Bagger, E. Poppitz and L. Randall, The R axion from dynamical supersymmetry breaking, Nucl. Phys. B 426 (1994) 3 [hep-ph/9405345] [SPIRES].

[23] M. Dine and J. Kehayias, Discrete R symmetries and low energy supersymmetry, arXiv:0909.1615 [SPIRES]. 